This item was submitted to Loughborough's Research Repository by the author.

Items in Figshare are protected by copyright, with all rights reserved, unless otherwise indicated.

\title{
Behaviour change determinants, the key to successful WASH strategies
}

PLEASE CITE THE PUBLISHED VERSION

PUBLISHER

(C) WEDC, Loughborough University

VERSION

VoR (Version of Record)

\section{PUBLISHER STATEMENT}

This work is made available according to the conditions of the Creative Commons Attribution-NonCommercialNoDerivatives 4.0 International (CC BY-NC-ND 4.0) licence. Full details of this licence are available at: https://creativecommons.org/licenses/by-nc-nd/4.0/

\section{LICENCE}

CC BY-NC-ND 4.0

\section{REPOSITORY RECORD}

Egreteau, Dany. 2019. "Behaviour Change Determinants, the Key to Successful WASH Strategies". figshare. https://hdl.handle.net/2134/31177. 
38th WEDC International Conference, Loughborough University, UK, 2015

\author{
WATER, SANITATION AND HYGIENE SERVICES BEYOND 2015: \\ IMPROVING ACCESS AND SUSTAINABILITY
}

\title{
Behaviour change determinants, the key to successful WASH strategies
}

\author{
D. Egreteau (France)
}

BRIEFING PAPER 2201

\begin{abstract}
The Approach focused on Behaviour Change Determinants $(A B C D)$ is a socio-anthropological inspired approach developed by SOLIDARITES INTERNATIONAL, based on an in-depth study of behaviors and their psychological, socio-cultural and environmental determinants in order to help professionals in the humanitarian and development fields through participative design of relevant and sustainable WASH interventions. This approach is integrated into the project cycle and can be developed through 3main stages: 1-Establishing a hierarchy of at-risk behaviours and practices; 2-Identifying the determinants of behaviours; 3-Developing the operational strategy. Although ABCD's methodological framework has been formalized, this framework remains flexible and can be adapted to different objectives for humanitarian and development projects, and throughout various intervention sectors (education, food security and livelihoods, etc.).
\end{abstract}

\section{Background}

Many organisations which are active in the WASH sector in humanitarian and development contexts are currently questioning the effectiveness of the assistance provided, with respect to the objective of reducing water-borne diseases (Hunter, 2011). WASH operations are often designed and evaluated in quantitative terms (e.g. number of water points and latrines installed). These results mask a very different reality in the field where many installations are seldom used or used improperly and are often not sustainable. The impact of WASH operations on the reduction of waterborne diseases is not very well known and there are probably many examples of operations which have worsened pre-existing situations (IRIN, 2013).

Installing or repairing infrastructure may not have the expected effects if the geographic, cultural and social realities of the targeted communities are not correctly taken into account (Mara, 2010). Indeed, though the installation of infrastructure should make the environment more conducive to appropriate WASH behaviours, it does not guarantee changes in behaviour in itself (Cairncross, 2004). This is where raising awareness about hygiene comes in, which is now recognized as a major part of WASH operations (Peal, 2010). However, awareness-raising activities are rarely integrated into operational strategies which focus primarily on technical choices and the installation of infrastructure. In addition, one-size-fits-all methodologies are repeated without taking the trouble to contextualize them. What is more, the great majority of awareness-raising activities are based on health messages focused on the dangers of "bad" behaviours rather than on the expected benefits of appropriate behaviours (Peal, 2010 \& Dreibelbis, 2013); it is assumed that once the population has been warned of the risk of contamination and the danger of waterborne diseases, they will adopt the thousand and one practices that are encouraged during these awarenessraising sessions. Here again, field realities are very different. The "good" practices which are promoted are restrictive and sometimes imply a major change in family habits. What is more, family habits are subject to a whole range of constraints and influences which are specific to their environment. This can sometimes facilitate changes in behaviour, but more often acts as a barrier to change.

The ABCD was developed in the light of the limits mentioned above. It is based on the work of the London School of Hygiene and Tropical Medicine (Aunger, in press) and the Swiss Federal Institute of Aquatic Science and Technology (Mosler, 2012) as well as the socio-anthropological aspects of different 
WASH project methodologies (e.g. FOAM, CLTS, participatory approaches and social marketing, Peal, 2010). ABCD attempts to bring these different sources together in an operational and pragmatic perspective. Its aim is to help develop operational strategies for WASH projects and to reinforce the coherence between infrastructure building, awareness raising and community mobilization activities. These strategies are based on the analysis of both WASH-related behaviours in the targeted community, and the determinants behind these behaviours. To date, the approach has been tested in four different contexts: two semi-permanent IDP camps in the Central African Republic, peri-urban communities in the Democratic Republic of Congo and rural communities in Bangladesh and Myanmar.

In this article, we will discuss the main principles of the $A B C D$ through the presentation of the 3 stages of the process supported by precise examples and lessons from the author's experience.

\section{Stage 1: Establishing a hierarchy of at risk behaviours and practices}

This Briefing Paper will focus on diarrhoeal diseases. However, the main principles of the approach are relevant for determining strategies in relation to any disease or malnutrition. As part of the ABCD, based on the available scientific knowledge (Curtis, 2003 \& Cairncross, 2010) and the experience of SOLIDARITES INTERNATIONAL, five key behaviours have been retained as having the most impact on diarrhoeal diseases:

1 Washing hands with soap at key moments of the day,

2 Adopting appropriate defecation practices,

3- Keeping latrines and house surroundings free of excrement,

4 Using potable water for drinking and cooking,

5- Collecting, transporting, storing and drinking water in an appropriate manner.

Scientific research has established that the risks of diarrhoeal diseases are reduced by $47 \%$ if communities have appropriate hand-washing practices. Sanitation programmes can reduce the risk of diarrhoea by up to $36 \%$ and operations aiming to improve the quantity and quality of water can reduce the risk of diarrhoea by $20 \%$ and $16 \%$ respectively. Though other behaviours can have an influence on the risk of diarrhoea (food hygiene, the management of organic waste, household hygiene...), the principle of the ABCD is to focus the available resources on the 5 above-mentioned behaviours as, in the majority of contexts, they will have the greatest impact on cases of diarrhoea.

An initial assessment phase establishes to what extent these behaviours are adopted (e.g. the percentage of the population who get their drinking and cooking water from potable water points). The initial assessment also analyses people's practices in relation to these behaviours, and particularly practices which could increase the risk of contamination. With regard to hand washing, for example, the assessment establishes whether hands are washed before meals or after going to the toilet, and also the way in which hands are washed: a common at-risk practice in communities in the north of the Central African Republic is to wash hands in a basin of water which is used collectively. Adults first dip their hands directly in the water, without soap, followed by all the other family members in order, from the oldest to the youngest. The last in line are children under 5 years of age, who are the most vulnerable to diarrhoeal diseases and the most exposed by this practice of collective hand-washing. Identifying this at-risk practice helps establish that although it is important to encourage hand-washing with soap, it is just as important to tackle this practice of collective hand-washing.

Once practices have been identified and the occurrence of behaviours has been established, the assessment aims to understand if there is a correlation between at-risk behaviours / practices and the frequency of cases of diarrhoea amongst children under the age of 5. For example, we find out whether there is a significant difference in the number of cases of diarrhoea between families who have drinking water storage containers that are closed in their houses ("doers", refers to families with appropriate practices) and families whose containers are not closed ("non-doers", refers to families with at-risk practices). The occurrence of behaviours, the qualitative analysis of practices and the established correlation between behaviours/practices and the number of cases of diarrhoea then make it possible to establish what behaviours and practices potentially have the most impact on diarrhoeal diseases. The operational strategy will thus focus on these behaviours and practices as a priority. 


\section{Stage 2: Identifying the determinants of behaviours}

Next, the ABCD aims to understand the positive and negative determinants of the prioritised behaviours and practices. For example, understanding what motivates families to have hygienic latrines and what may prevent other families from maintaining them properly. There are two distinct groups of determinants: external factors linked to the environment and factors which are internal to the operational strategy target groups (generally mothers).

\section{Environmental determinants}

Environmental determinants include, in an initial circle, all the aspects linked to the family: decision-making power, management of the family budget, authority, the respective responsibilities of different family members, the distribution of WASH tasks, childcare, etc. A second circle includes aspects from the community, village or neighbourhood environment: accessibility to water points, health centres, communication channels, demographics, ethnic and religious factors, opinion-makers, livelihoods, accessibility to markets, etc. The final circle includes regional and national environmental aspects: public policies in terms of Health, Education, WASH, Security, exposure to natural disasters, cultural systems, etc.

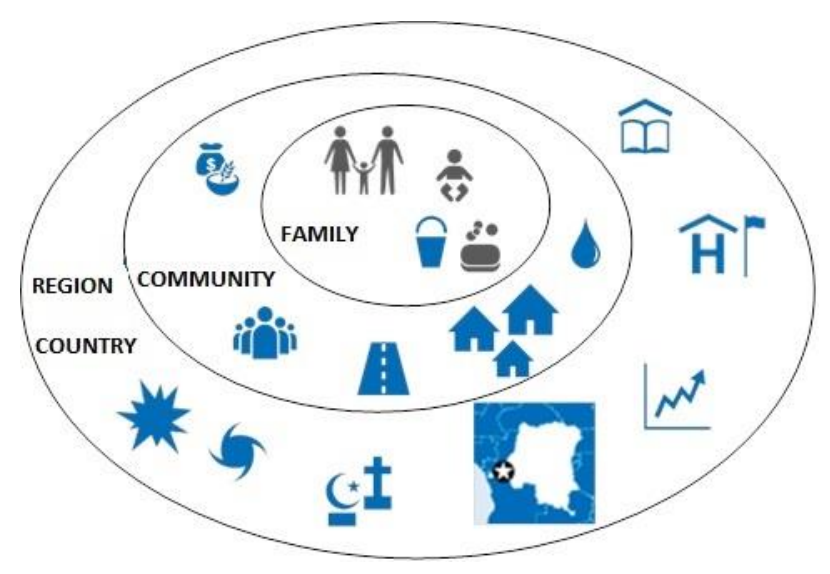

Figure 1. Environmental determinants

Source: Solidarites International

\section{Internal determinants}

Internal determinants are the result of the psychological and physical characteristics of individuals. We try to establish which of these help or hinder individuals in terms of adopting appropriate behaviours. These positive or negative motivations can be economic, religious, based on security, prestige, privacy, disgust, comfort, maternal love, seduction, etc. The ABCD also aims to identify to what extent individuals know the causes of diarrhoeal diseases and how they perceive the severity of these diseases for their young children and the consequences of diarrhoea on the rest of the family. However, the approach is based on the principle that though knowledge of the disease and its severity can favour the adoption of appropriate practices, it is not in itself sufficient to encourage a change in behaviour, due to the greater influence of the other abovementioned motivators and barriers (Aunger, in press). The ABCD also explores the extent to which individuals feel capable of regularly repeating behaviours (perceived capacity) and the influence of their relatives, friends and neighbours (injunctive norm). The approach can also look at physical determinants, such as questions of accessibility and physical effort related to WASH practices.

Finally, the assessment tries to identify the most significant determinants with respect to the prioritised practices and behaviours and evaluates the extent to which these determinants exist amongst doers and nondoers. If we establish that there is in fact no significant difference between the two groups for a given determinant, it is presumed that acting on this determinant will have little impact on the behaviour of nondoers. However, these results cannot be used without a complementary qualitative analysis, notably due to the interaction, which is difficult to quantify, between 1-behaviours with each other and 2-determinants with 
each other. For example, if it appears that families who maintain their latrines in a hygienic manner and those who do not all have an accurate idea of the seriousness and the causes of diarrhoea (here, faeces), it is possible to conclude that classic awareness-raising focused on health will probably have little impact on encouraging non-doer families to keep their latrines in a better state. On the other hand, if we observe that the prestige of having clean latrines is mentioned much more by the doers, we deduce that prestige can be one of the focuses of awareness-raising content in order to incite the non-doers to adopt appropriate latrine cleaning practices. For example, the following was regularly heard in Kinshasa: "It is important to have clean latrines so that visitors see that we are a 'good' family".

\section{Stage 3: Developing the operational strategy}

The operational strategy will be based on the behaviours and practices which can potentially have the most impact on reducing the occurrence of diarrhoea amongst children under 5 years of age. Analysis of environmental determinants essentially allows barriers linked to the context where people live to be identified (e.g. availability of water, difficulties with communication channels, intercommunity tension, insecurity, livelihoods which do not cover basic needs) and to base technical decisions and operations on these.

For example, if there are tensions between different communities (castes) in a displaced persons' camp in India, installing a single water point in the area occupied by one of the castes will mean that it will be underused by the other caste. Understanding these social tensions and other environmental determinants will make it possible to choose appropriate sites and technical solutions (in this case, by installing a water point for each community). Analysis of the environment will also make it possible to identify the people who have the most influence over the targeted group, both inside families and in the community. For example, conducting awareness-raising activities with mothers who do not have much authority or freedom of expression within their husbands' families (generally the case in India, Bangladesh or in the north of the Central African Republic) would be useless if the husband's family prevents the mother from adopting appropriate practices. In cases like this, awareness campaigns should target both mothers and mothers-inlaw (in reality, women between 18 and 45 years old). Detailed understanding of the social environment will allow opinion leaders, potential awareness-raising relays and the most pertinent community mobilization strategies to be identified.

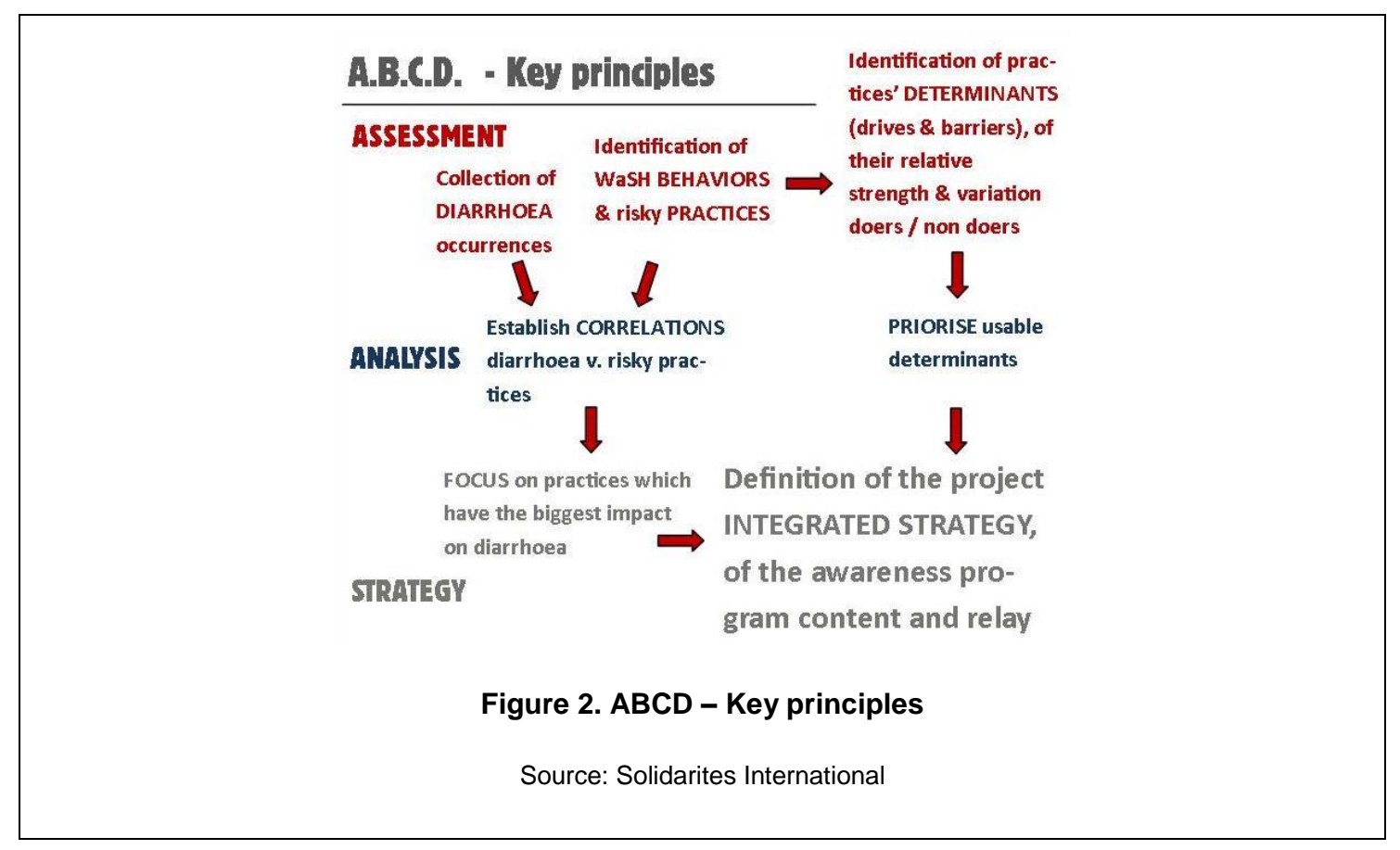

Analysis of internal determinants can have an impact on the choice of technical options. For example, taste is often an essential determinant in the choice of water points for drinking. Thus, installing a pump which draws water from a ferruginous aquifer can lead to the water point being under-used and even 
abandoned even though the water is potable. Other technical solutions should be given priority (treatment of surface water or reaching aquifers with neutral tasting water). Analysis of internal determinants is also essential to produce awareness-raising and community mobilization messages - c.f. the example of prestige given above as a way of encouraging the maintenance of toilets.

\section{Conclusion and recommendations}

The ABCD is based on the targeted communities' environment and day-to-day lives. On the one hand, it encourages observation and critical analysis on the part of the local team in charge of collecting information, and on the other hand, it forces us to think about the context of the project in order to develop integrated and custom-made operational strategies. The ABCD aims to be an adaptable rather than a rigid project methodology. Though its main principles are relevant in any context (training and mobilization of teams; focus on at-risk behaviours; analysis of practices, external and internal determinants, and integrated strategies; support and monitoring of practices), it is flexible and can be adapted depending on the environment, resources and progress of the project. The ABCD demands a time commitment of roughly one month, a significant investment in terms of resources needed to carry out the study and the development of an operation strategy in collaboration with the field team. However, the principles of the approach are easily tested and can be adapted to fit the existing tools and methodologies of an individual organisation. Furthermore, it can be implemented by any Project Manager who wants to establish a framework for the sustainable adoption of appropriate sanitation and hygiene practices.

Field teams tend to be receptive to the ABCD due to the fact that they already employ non-health based strategies when convincing communities to adopt appropriate behaviours. They have a greater comprehension of the environmental determinants (e.g. magic, culture, family habits, etc.) and therefore, hold an informal expertise on behaviour determinants, which is important to highlight. This can be done through a preliminary training during which basic principles of the approach are explained. Field team members are encouraged, while implementing the quantitative surveys, to provide feedback on what they observe during household visits. Not only do they bring back precious qualitative information but they gain a sense of empowerment, becoming more committed to the implementation phase and seeking to build awareness based on the most powerful determinants identified during the assessment. It is often more difficult to engage WASH managers (local or expatriates) to take ownership of the approach. In order to get a relevant understanding of behaviour determinants through the ABCD it is essential to take the time for qualitative analysis and to work in a participatory manner with field staff members. Managers may be subject to budget and time constraints that divert them from appreciating the importance of such a survey. Furthermore, mangers with a strong technical background do not necessarily place a great deal of importance on the socio-cultural aspects of their project.

It is therefore important that 1 ) at the project design stage, resources and time are properly allocated to account for implementation of the ABCD, 2) the NGO encourages managers to move away from a purely technical approach to WASH and pay strong attention to the socio-cultural context in which their projects operate, 3 ) a one or two-day training course is organized at the beginning of the project on the ABCD basics ( 5 key behaviours, the environmental and internal determinants).

From a methodological perspective, it is important to emphasize that, once the team recognises the necessity of understanding practices as well as the determinants of these practices, the ABCD can be implemented using the tools and methodologies with which the agency, manager and field teams are accustomed. Successful implementation of the ABCD relies, above all, on managers and local teams adopting a positive attitude towards behaviour change socio-anthropological basics.

\section{References}

Aunger R, Curtis V. The Evo - Eco Approach to Behaviour Change. Applied Evolutionary Anthropology. Springer Verlag. In press.

Coombes Y. \& Devine J. Introducing FOAM (2010): A framework to Analyse Handwashing Behaviors to Design Effective Handwashing Programs, WSP.

Cairncross S, Shordt K. (2004): It does last! Some findings from a multi-country study of hygiene sustainability. Waterlines.

Cairncross S, Shordt K, Zacharia S, Govindan BK (2005): What causes sustainable changes in hygiene behaviour? A cross-sectional study from Kerala, India. Soc Sci Med. 
Cairncross S et al. (2010): Water, sanitation and hygiene for the prevention of diarrhoea, International Journal of Epidemiology, 39 (suppl 1).

Curtis V, Danquah L, Aunger R. (2009): Planned, motivated and habitual hygiene behaviour: an eleven country review. Health Educ Res. 2009;4: 655-673.

Curtis V. Talking Dirty (2003): How to save a million lives. International Journal of Environmental Health Research. 2003 Jun;13 Suppl 1:S73-9.

Dreibelbis Robert et al.(2013), «The Integrated Behavioural Model for Water, Sanitation, and Hygiene: a systematic review of behavioural models and a framework for designing and evaluating behaviour change interventions in infrastructure-restricted settings », BMC Public Health, 13 (1), Dec 2013, pp. 113.

Hunter P. (2010): Waterborne diseases and water safety. in CRASH/Fondation - Médecins Sans Frontières. Is humanitarian water safe to drink?

IRIN (2014): Analysis: Sanitizing the Truth - When WASH Fails. Africa English Service. Al Bawaba (Middle East).Ltd. 2013. HighBeam Research.

Mara D, Lane J, Scott B, Trouba D. (2010): Sanitation and health. PLoS Med.

Mosler HJ. (2012): A systematic approach to behavior change, interventions for the water and sanitation sector in developing countries: a conceptual model, a review, and a guideline, International Journal of Environmental Health Research.

National Institute of Statistics and UNICEF (2011): Multiple Indicator Cluster Survey, Democratic Republic of Congo (MICS-RDC 2010). Final Report.

Peal et al (2010): Hygiene and Sanitation Software, An Overview of Approaches. Water Supply \& Sanitation Collaborative Council, Geneva, Switzerland.

WHO (2008): Guidelines for Drinking Water Quality. Geneva, p.20.

\section{Note}

The author formerly worked as a Head of Mission for SOLIDARITES INTERNATIONAL in Bangladesh, and has been conducting R\&D missions on the subject of behavioural approaches as part of SOLIDARITES INTERNATIONAL's WASH programmes.

\section{Contact details}

Dany Egreteau

Solidarites International

89, rue de Paris - 92110 Clichy - France

Tel: +33 (0) 1.76.21.86.00

Email: dany.egreteau@gmail.com

www.solidarites.org 